\title{
microRNA-182 inhibits the proliferation and migration of glioma cells through the induction of neuritin expression
}

\author{
$\mathrm{YAFENG}^{1}$, TE LIU ${ }^{1,2}$ and YUNCHENG WU ${ }^{1}$ \\ ${ }^{1}$ Department of Neurology, Shanghai First People's Hospital, Shanghai Jiao Tong University School of Medicine, \\ Shanghai 200080; ${ }^{2}$ Department of Traditional Chinese Medicine, Shanghai Geriatric Institute of Chinese Medicine, \\ Longhua Hospital, Shanghai University of Traditional Chinese Medicine, Shanghai 200031, P.R. China
}

Received August 27, 2014; Accepted May 20, 2015

DOI: $10.3892 / \mathrm{ol} .2015 .3365$

\begin{abstract}
Astrocytomas are the most common type of glial tumors and carry a poor prognosis. However, the pathogenesis of astrocytomas remains to be elucidated. Neuritin, a novel member of the neurotrophic factors family, has been shown to be associated with tumor malignancy, via the regulation of apoptosis and proliferation. In the present study, microRNA-182 (miR-182) was cloned and transfected into the U251 human astrocytoma cell line, in order to investigate its regulatory effects on the proliferation and migration of these cells, as well as its association with the expression of neuritin. The results showed that miR-182 specifically targets the gene encoding neuritin, NRN1, as demonstrated by a reduction in the protein and mRNA levels of NRN1. In addition, overexpression of miR-182 affected cell cycle regulation and cell migration capacity in vitro, which may have been associated with the promotion of apoptosis by this molecule. In conclusion, endogenous miR-182 may be involved in the pathogenesis of astrocytoma, which is associated with the miR-182-regulated gene, NRN1.
\end{abstract}

Correspondence to: Professor Yuncheng Wu, Department of Neurology, Shanghai First People's Hospital, Shanghai Jiao Tong University School of Medicine, 100 Haining Road, Shanghai 200080, P.R. China

E-mail: yunchw@medmail.com.cn

Professor Te Liu, Department of Traditional Chinese Medicine, Shanghai Geriatric Institute of Chinese Medicine, Longhua Hospital, Shanghai University of Traditional Chinese Medicine, 365 South Xiangyang Road, Shanghai 200031, P.R. China E-mail: teliu79@126.com

Abbreviations: CCK8, cell counting kit-8; FBS, fetal bovine serum; FCM, flow cytometric; miRNA, microRNA; NF, neurotrophic factor; NSCLC, non-small cell lung cancer; pre-miRNA, precursor miRNA

Key words: astrocytoma, microRNA-182, neuritin, proliferation, migration

\section{Introduction}

Gliomas account for $\sim 81 \%$ of malignant intracranial tumors, among which astrocytomas are the most common type (1). A recent study indicated that $14.5 \%$ of patients with astrocytoma survived for five years from diagnosis (2). However, the pathogenesis and progression of astrocytoma remain poorly understood, and therapeutic strategies for this disease are limited. Due to the necessity to identify novel therapeutic approaches, further studies are required, in order to examine the biological processes underlying the development of astrocytoma (3).

microRNAs (miRNAs) are small, non-coding RNA molecules of 20-23 nucleotides in length. They have been shown to be involved in regulating the development and maintenance of undifferentiated or incompletely differentiated cell types (4-6). Accumulating evidence indicates that dysregulation of specific miRNAs may be associated with certain types of cancer, and that miRNAs act as oncogenes or tumor suppressors of particular target genes (7-10). miR-155, for example, acts as an inhibitor of ovarian cancer-initiating cells by regulating claudin 1, thus disrupting the development of human ovarian cancer (11). Furthermore, fragments of miRNA have been detected and measured in the serum of patients with cancer, demonstrating that they may be useful as biomarkers for disease diagnosis. One such example is the upregulation of miR-23a, 27a and 24 in the serum of patients with hepatocellular carcinoma, which are associated with tumor-suppressive activities (12). However, whether specific miRNAs are dysregulated in astrocytoma, and whether they contribute to the development and progression of this disease remains unclear.

Neuritin, encoded by the NRN1 gene, is a novel member of the neurotrophic factor (NF) family, and is induced by neuronal activity in the rat hippocampus (13). Within the central nervous system, neuritin promotes neurite outgrowth and synaptic maturation, protects motor neuron axons and regulates apoptosis of proliferative neurons (13). Notably, neuritin has also been shown to be expressed outside the nervous system, including in invasive breast carcinoma and Kaposi's sarcoma, where it is associated with apoptosis and tumorigenesis $(14,15)$. In accordance with the results of previous studies, Zhang et al (16) demonstrated that neuritin 
was overexpressed in the U251 human astrocytoma cell line, and that the level of expression was positively correlated with tumor malignancy. Furthermore, recent studies have demonstrated that neuritin acts as a novel angiogenic factor, promoting tumor angiogenesis (17), and that it modulates neuronal migration (18). However, the characteristics and mechanism of action of neuritin regulation in astrocytomas, particularly at the epigenetic level, have not yet been described.

The present study investigated whether miR-182 specifically targets NRN1, and influences cell proliferation and migration ability in the U251 human astrocytoma cell line.

\section{Materials and methods}

Bioinformatics analysis of miR-182 and the 3'-UTR of NRN1. The precursor miRNA (pre-miRNA) sequences, mature miRNA sequences, chromosomal locations, and the length of miR-182 and the target gene, NRN1, were analyzed in multiple species (human, rhesus and mouse) using the online research tool of the miRBase Target database (http://www.mirbase.org) $(19,20)$.

Cell culture. The U251 human astrocytoma cell line was grown in Dulbecco's modified Eagle's medium (DMEM; Hyclone, Logan, UT, USA), supplemented with $10 \%$ fetal bovine serum (FBS; PAA Lab., Inc., Morningside, Queensland, Australia), penicillin $(100 \mathrm{U} / \mathrm{ml})$, streptomycin $(100 \mathrm{U} / \mathrm{ml})$ and $2 \mathrm{mM}$ L-glutamine (Hyclone). U251 cells were maintained at $37^{\circ} \mathrm{C}$ in a humidified atmosphere of air containing $5 \% \mathrm{CO}_{2}$.

Vector construction. For experiments measuring miRNA-182 expression, the plasmid pRNAT-CMV32-cGFP-mir182 (pre-miRNA of miRNA-182), oligonucleotide pairs for the pre-miRNA of miRNA-182 and linker sequences with BamHI and XhoI sites, were chemically synthesized (20). The sequences of the oligonucleotides were as follows: Top strand, 5'-GTggatccCTGTTTGGCAATGGTAGAACTCACACTT TTTGCCTCCAACTGACTCCTACATATTAGCATTAACAGc tcgagCC-3' and bottom strand, 5'-GGctcgagCTGTTAATGC TAATATGTAGGAGTCAGTTGGAGGCAAAAAAGTGTG AGTTCTACCATTGCCAAACAGggatccAC-3' (sequences corresponding to miRNA-182 seed sequences are shown in bold and restriction enzyme sites are in lower case). In order to build the expression plasmid, the pairs of oligonucleotides were annealed and inserted into the multiple cloning sites between the BamHI and XhoI restriction sites in the pRNAT-CMV32-cGFP/Neo vector (GenScript,Piscataway, NJ, USA). The negative control plasmid, pRNAT-CMV32-cGFPmir182-Mut, was similarly constructed, with 17 nucleotides in the sequence corresponding to the miRNA-182 seed sequence, mutated from TTTGGCAATGGTAGAAC to TaTGcgAtTGGaAcAtg (mutations shown in lower case). Transfection of $0.3 \mu \mathrm{g}$ of miRNA-182 or miRNA-182 mutant expression vector into the U251 cell line was conducted using Lipofectamine 2000 ${ }^{\mathrm{TM}}$ (Thermo Fisher Scientific, Inc.,Waltham, MA, USA) transfection reagent, according to the manufacturer's instructions.

RNA extraction and analysis by reverse transcription-quantitative polymerase chain reaction $(R T-q P C R)$. Total RNA from each group of cells was isolated using TRIzol ${ }^{\mathrm{TM}}$ reagent
(Invitrogen Life Technologies, Carlsbad, CA, USA) according to the manufacturer's instructions. RNA samples were treated with DNase I (Sigma-Aldrich, St. Louis, MO, USA), quantified and then reverse-transcribed into cDNA using the ReverTra Ace- $\alpha$ first strand cDNA synthesis kit (Toyobo Co., Ltd., Osaka, Japan). RT-qPCR was conducted using a RealPlex4 real-time PCR detection system (Eppendorf Co., Ltd., Hamburg, Germany), with SYBR-Green real-time PCR Master Mix (Toyobo Co., Ltd.) as the detection dye. The following PCR primer sequences used: Forward, 5'-ATCCTCGCGGTGCAAATAG-3' and reverse, 5'-GAGCAAACAGTCCGAAAAGC-3' for NRN1 and forward, 5'-CAGCCACCCGAGATTGAGCA-3' and reverse, 5'-TAGTAGCGACGGGCGGTGTG-3' for 18S rRNA. PCR was performed under the following conditions: Denaturation at $95^{\circ} \mathrm{C}$ for $15 \mathrm{sec}$, annealing at $58^{\circ} \mathrm{C}$ for $30 \mathrm{sec}$ and extension at $72^{\circ} \mathrm{C}$ for $42 \mathrm{sec}$. The comparative threshold cycle $(\mathrm{Ct})$ was used to determine relative NRN1 gene expression, normalized to that of $18 \mathrm{~S}$ rRNA. For each sample, the $\mathrm{Ct}$ values of the genes were normalized using the formula $\Delta \mathrm{Ct}=\mathrm{Ct}_{\text {genes }}-\mathrm{Ct}_{18 \mathrm{~s} \text { rRNA }}$. In order to determine relative expression levels, the following formula was used: $\Delta \Delta \mathrm{Ct}=\Delta \mathrm{Ct}_{\text {all groups }}-\Delta \mathrm{Ct}_{\text {blank control group }}$. The values used to plot the relative expression of markers were calculated using the expression $2^{-\Delta \Delta \mathrm{Ct}}$. The cDNA of each gene was amplified with primers as previously described (21).

Flow cytometric (FCM) analysis of cell cycle progression. Each group of U251 cells was seeded at $3 \times 10^{5}$ cells per well in six-well plates, and cultured until $85 \%$ confluence was reached. After washing with phosphate-buffered saline (PBS) three times, cells were collected by centrifugation (Allegra X-22R; Beckman Coulter, Brea, CA, USA) at $1000 \mathrm{x}$ g for $5 \mathrm{~min}$. Cell pellets were resuspended in $1 \mathrm{ml}$ of PBS, fixed in $75 \%$ ice-cold ethanol, and stored in a freezer for $>48 \mathrm{~h}$. Prior to FCM analysis, fixed cells were centrifuged and washed twice with PBS, then resuspended in propidium iodide (PI) staining solution (Sigma-Aldrich), containing $50 \mu \mathrm{l} / \mathrm{ml} \mathrm{PI}$ and $250 \mu \mathrm{l} / \mathrm{ml}$ RNase A (Sigma-Aldrich, Carlsbad, CA, USA). The cell suspension was incubated for 30 min at $4^{\circ} \mathrm{C}$ in darkness and analyzed using FACS (FCM-500; Beckman Coulter). A total of 20,000 events were acquired for the analysis, using CellQuest software (version 5.1; BD Biosciences, Franklin Lakes, NJ, USA).

Luciferase reporter assay. All steps of the luciferase reporter assay were performed, as previously described $(12,22)$. Non-anthropogenic NIH-3T3 cells (Stem Cell Bank, Chinese Academy of Sciences, Shanghai, China) were used to avoid the influence of endogenous human miRNA-182. NIH-3T3 cells were seeded at $3 \times 10^{4}$ cells per well in 48-well plates and cotransfected with $400 \mathrm{ng}$ pRNAT-CMV32-cGFP-mir182, pRNAT-CMV32-cGFP or pRNAT-CMV32-cGFP-mir182-mut, and $20 \mathrm{ng}$ pGL3cm-CTTN-3UTR-WT or pGL3cm-CTTN-3UTR-mut, and pGL-3 (Promega Corporation, Madison, WI, USA), using Lipofectamine 2000 transfection reagent according to the manufacturer's instructions. Following transfection for $48 \mathrm{~h}$, the luciferase activity was measured using the dual-luciferase reporter assay system (Promega Corporation).

Northern blotting. All steps for the northern blotting analysis were performed as previously described (18). For all groups, 

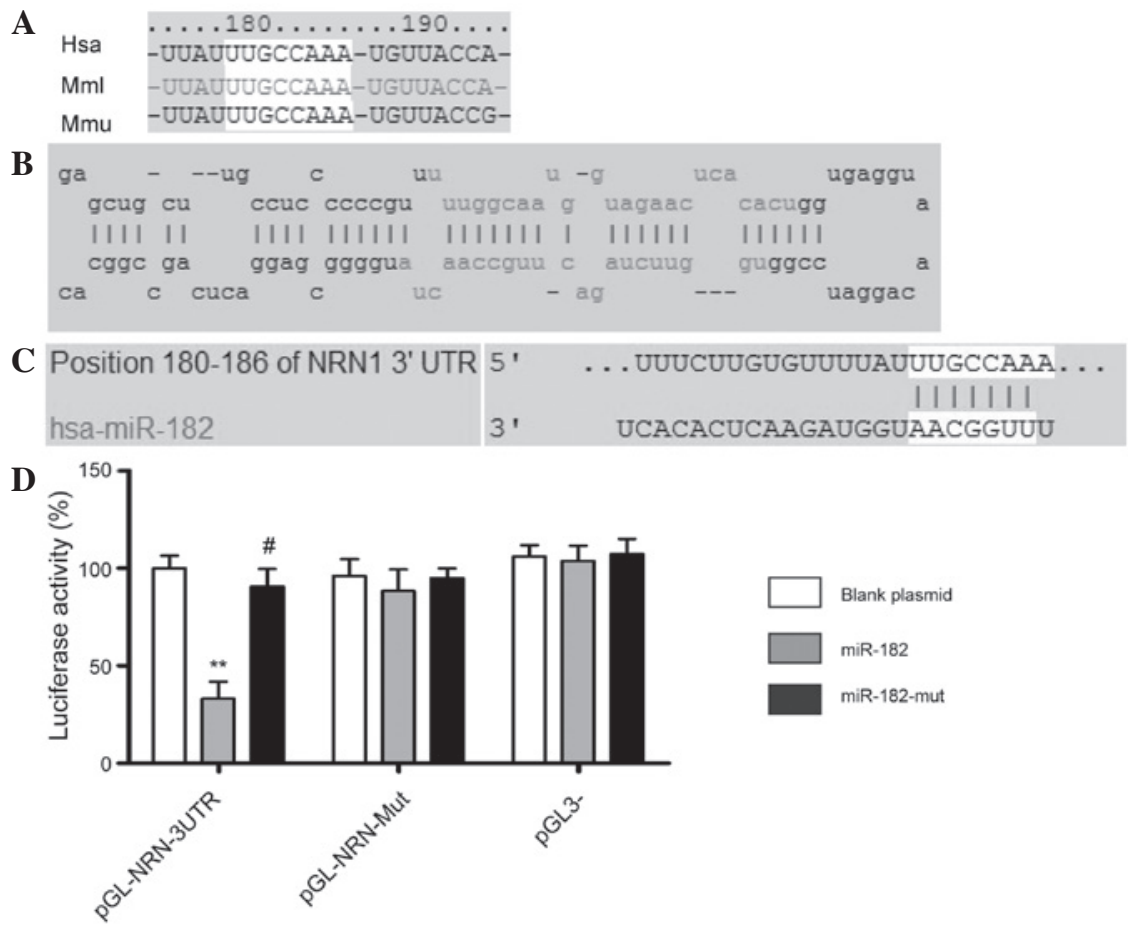

Figure 1.3'-UTR of human NRN1 mRNA contains miR-182 binding sites. (A) The mature miR-182 sequences of Has (human), Mml (rhesus) and Mmu (mouse) were analyzed, using bioinformatics tools. (B) Secondary structure of miR-182 with stem-loop. (C) Complementarity between miR-182 and the putative human NRN1 3'UTR site target (180-186 bp downstream). (D) Luciferase activity assay. ${ }^{* *} \mathrm{P}<0.01$ and ${ }^{\text {" }} \mathrm{P}>0.05$ vs. control plasmid. $\mathrm{n}=3$. UTR, untranslated region; NRN1, neuritin 1; miR, microRNA.

$20 \mu \mathrm{g}$ of high-quality total RNA was analyzed on a $7.5 \mathrm{M}$ urea, $12 \%$ paraformaldehyde denaturing gel, and transferred to a Hybond- $\mathrm{N}^{+}$nylon membrane (Amersham, Freiburg, Germany). Membranes were cross-linked using UV light for 30s at $1200 \mathrm{~mJ} / \mathrm{m}^{2}$. Hybridization was performed with the miRNA-182 antisense StarFire probe, 5'-TTTGGCAATGGTAGAACTCACACT-3' (IDT, Coralville, IA, USA), in order to detect the 22-nucleotide miRNA-182 fragments, according to the manufacturer's instructions. After washing, membranes were exposed for 20-40 h to Kodak XAR-5 film (Sigma-Aldrich). As a positive control, all membranes were hybridized with a human U6 snRNA probe, 5'-GCAGGGGCC ATGCTAATCTTCTCTGTATCG-3'. Exposure times for the U6 control probe varied from between 15 to $30 \mathrm{~min}$.

Western blotting. Total protein extracts of each group of cells were resolved by $12 \%$ SDS-PAGE and transferred to PVDF membranes (IPVH00010; Merck Millipore, Billerica, MA, USA). After blocking, the PVDF membranes were washed 4 times for 15 min with Tris-buffered saline with Tween-20 ${ }^{\circledR}$ (TBST; Beyotime Institute of Biotechnology, Haimen, China) at room temperature and incubated with rabbit anti-human neuritin polyclonal antibody (1:500 dilution; cat no. Ab64186; Abcam, San Francisco, CA, USA). Following extensive washing, membranes were incubated with the secondary peroxidase-linked goat anti-rabbit IgG (1:1000 dilution; cat no. sc-45101; Santa Cruz Biotechnology, Inc., Dallas, TX, USA) for $1 \mathrm{~h}$. After washing four times for $15 \mathrm{~min}$ with TBST at room temperature, immunoreactivity was visualized using the enhanced chemiluminescence ECL kit (Pierce Biotechnology, Inc., Rockford, IL, USA), and membranes were exposed to Kodak XAR-5 film.
Transwell migration assay. All steps were performed as previously described (23). Cells $\left(2 \times 10^{5}\right)$ were resuspended in $200 \mu \mathrm{l}$ of serum-free medium, and seeded on the top chamber of the 8.0- $\mu \mathrm{m}$ pore, $6.5-\mathrm{mm}$ polycarbonate transwell filters (Corning, Lowell, MA, USA). Complete medium (600 $\mu \mathrm{l})$ containing $10 \%$ FBS was added to the bottom chamber. For the invasion assay, inserts coated with Matrigel ${ }^{\mathrm{TM}}$ (product no. 356234; Shanghai Hengyuan Macromolecular Materials Co., Ltd., Shanghai, China) were used. Cells were allowed to migrate for 24,48 or $72 \mathrm{~h}$ at $37^{\circ} \mathrm{C}$ in a humidified incubator with $5 \% \mathrm{CO}_{2}$. The cells that attached to the lower surface of the membranes were fixed in $4 \%$ paraformaldehyde at room temperature for 30 min and stained with DAPI (C1002; Beyotime Institute of Biotechnology), and the number of cells on the lower surface of the filter was counted under the microscope (BX51TF; Olympus Corporation, Tokyo, Japan). A total of five fields were counted from each transwell filter.

Cell Counting kit-8 (CCK8) cell viability assay. Cell viability was assessed using a CCK8 assay (Dojindo, Kumamoto, Japan). U251 cells transfected with miR-182 or mutant miR-182, were seeded into 96-well plates. Following transfection for 0 and $72 \mathrm{~h}, 10 \mu \mathrm{l}$ of CCK8 was added to each well and incubated at $37^{\circ} \mathrm{C}$ for $1 \mathrm{~h}$. Cell viability was measured at $450 \mathrm{~nm}$ using an ELISA reader (BioTek, Winooski, VT, USA), according to the manufacturer's instructions.

Statistical analysis. Each experiment was performed at least three times, and data are presented as the mean \pm standard error, where applicable. Differences were evaluated using Student's t-test. $\mathrm{P}<0.05$ was considered to indicate a statistically significant difference. 

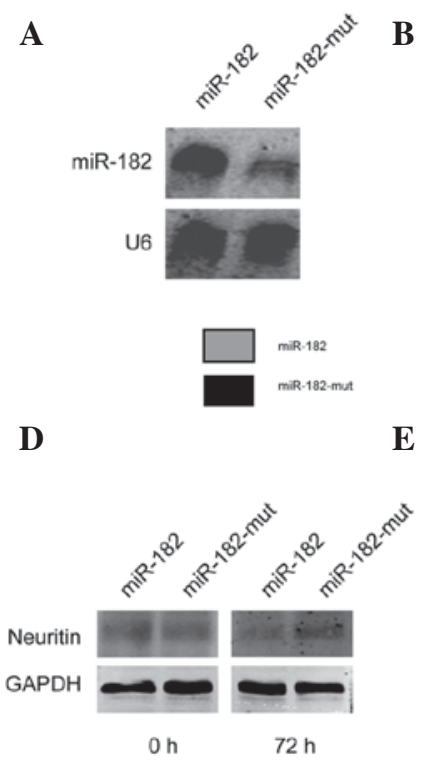

B
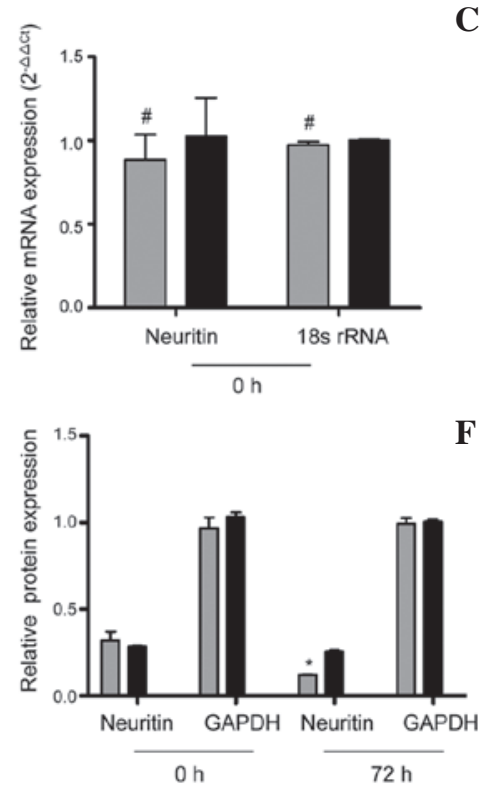

C
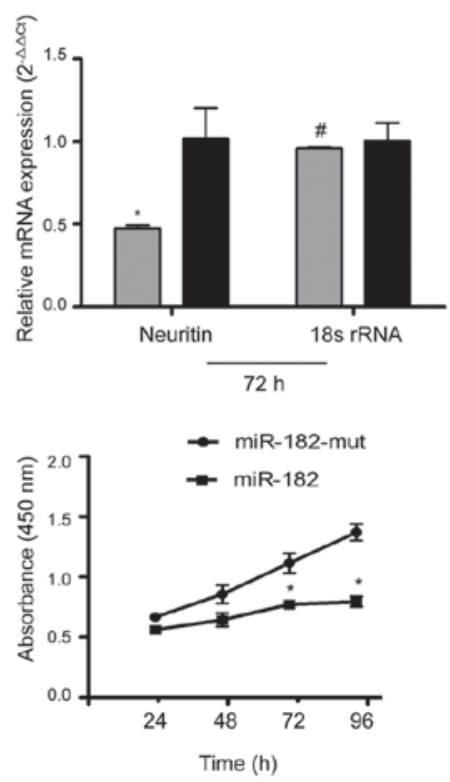

Figure 2. miR-182 regulated NRN1 expression and altered cell proliferation. (A) Northern blotting demonstrated a difference in the hybridization signal. The human U6 probe was used as a loading control. (B) and (C) reverse transcription-quantitative polymerase chain reaction analysis, and (D) and (E) western blotting analysis of neuritin mRNA and protein expression, respectively, within the different groups. (F) A CCK8 assay was used to determine cell survival. ${ }^{*} \mathrm{P}<0.05$ and ${ }^{\#} \mathrm{P}>0.05$ vs. mutant miR-182-transfected cells. $\mathrm{n}=3$. miR, microRNA; NRN1, neuritin 1.

\section{Results}

Bioinformatics analysis of miR-182 and the 3'-UTR of NRN1. Multi-species bioinformatics was performed using the online research tool of the miRBase Target database (http://www.mirbase.org) $(19,20)$. The present study focused on human miR-182, which may target the 3'-UTR of NRN1. This site is conserved, to varying degrees, across different species (Fig. 1).

Identification of miR-182 binding sites in the 3'-UTR region of NRN1. A luciferase reporter assay was used to determine whether NRN1 expression was regulated by mature miR-182. Plasmids with a WT or mutant NRN1 mRNA 3'-UTR miR-182 binding site, or an empty plasmid control were cotransfected with the miR-182 expression vector (WT miR-182, empty vector or mutant miR-182) into NIH-3T3 cells. The results showed that the activity of the luciferase reporter containing the WT NRN1 3'-UTR, was significantly inhibited following transfection with WT miR-182, while the luciferase activity of the reporter gene with the mutated NRN1 3'-UTR was unchanged, indicating that miR-182 may target NRN1 mRNA by specifically binding to its 3'-UTR (Fig. 1D).

miR-182 influences the expression of neuritin in the U251 human astrocytoma cell line. Subsequently, the regulation of neuritin expression in U251 cells by exogenous miR-182 was examined. Northern blotting results showed that the hybridization signal of mutant miR-182-transfected U251 cells was weaker than that in the miR-182-transfected cells (Fig. 2A). These results were confirmed by RT-qPCR, which showed that at $72 \mathrm{~h}$ following transfection with the miR-182 expression vector, NRN1 mRNA levels decreased significantly, compared with those of U251 cells transfected with mutant miR-182. The relative mRNA expression is shown following normalization to that of $18 \mathrm{~S}$ rRNA, which was used as an internal control (Fig. 2B and C). Furthermore, western blotting indicated that protein levels of neuritin in mutant miR-182-transfected cells were $\sim 2$-fold higher than levels in cells transfected with miR-182 (Fig. 2D and E). The CCK8 cell viability assay indicated no difference in viability between miR-182-transfected cells and mutant miR-182-transfected cells at $24 \mathrm{~h}$. However, a significant reduction in cell viability was observed in cells transfected with miR-182 at $72 \mathrm{~h}$ and $96 \mathrm{~h}$, compared with mutant miR-182-transfected cells (Fig. 2F). These data suggest that exogenous miR-182 downregulates NRN1 expression.

Alteration of cell cycle progression and invasion ability of U251 cells following miR-182 transfection. A flow cytometric (FCM) assay was performed in order to detect changes in cell cycle progression. miR-182-transfected and mutant miR-182-transfected U251 cells were stained with PI and analyzed by FCM. The results showed no significant differences in cell cycle distribution between miR-182-transfected and mutant miR-182-transfected cells at $0 \mathrm{~h}$ (Fig. 3A). However, at $72 \mathrm{~h}$ following transfection, the number of cells that were arrested in $\mathrm{S}$ phase of the cell cycle, and the percentage of cells in the G2/M phase, significantly decreased in the miR-182-transfected cells compared with the proportions in the miR-182-mut-transfected cells (Fig. 3A-C). These results indicate that increased expression of miR-182 significantly affects cell cycle progression in vitro. The transwell migration invasion assay showed that the number of invading cells was significantly reduced in miR-182-transfected cells at $48 \mathrm{~h}$ and $72 \mathrm{~h}$, compared with the number of invading mutant miR-182-transfected cells, while no significant difference in the number of invading cells was detected between the two groups $24 \mathrm{~h}$ after transfection (Fig. 3D and E). These results suggested that reduced 
A

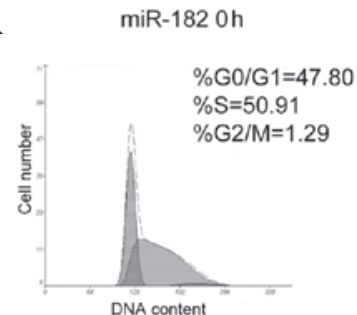

$\operatorname{miR}-18272 \mathrm{~h}$

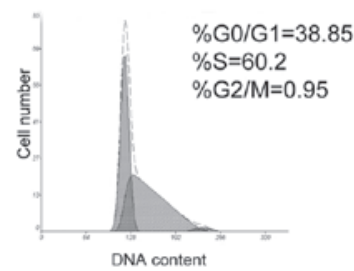

D

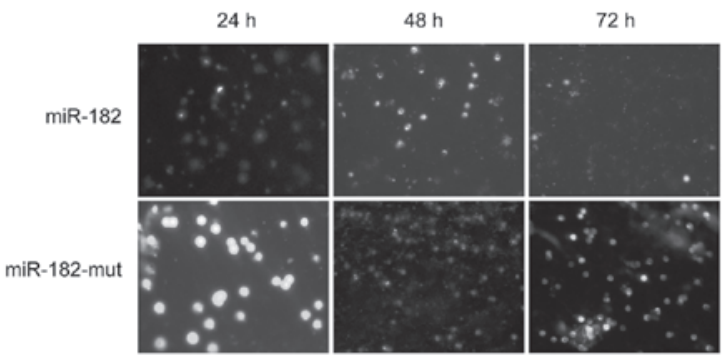

B

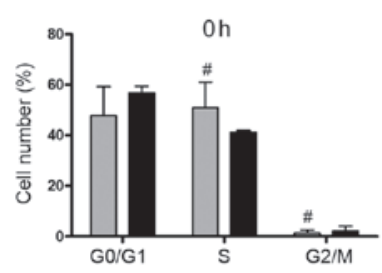

C
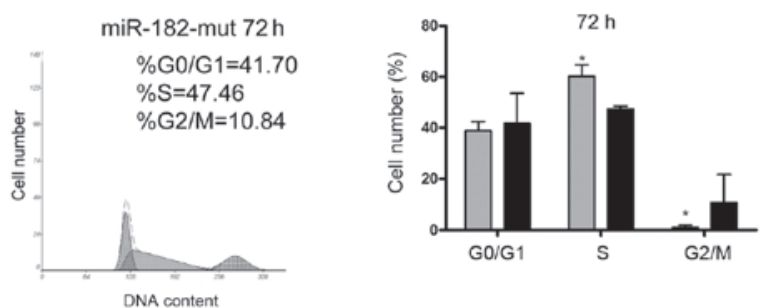

$\mathbf{E}$
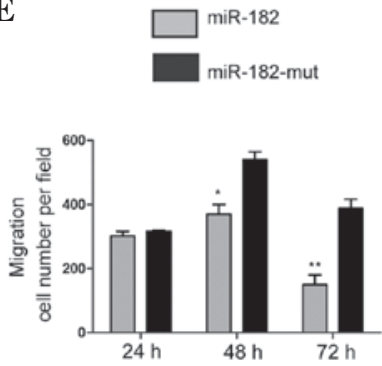

Figure 3. Exogenous miR-182 influenced cell cycle progression and the invasion ability of U251 cells. (A-C) Flow cytometry demonstrated changes in cell cycle progression between groups. (D) and (E) A transwell migration invasion assay indicated the alteration of cell invasiveness following transfection. ${ }^{*} \mathrm{P}<0.05$, ${ }^{* *} \mathrm{P}<0.01$ and ${ }^{\#} \mathrm{P}>0.05$ vs. mutant miR-182-transfected cells. $\mathrm{n}=3$. miR, microRNA.

A
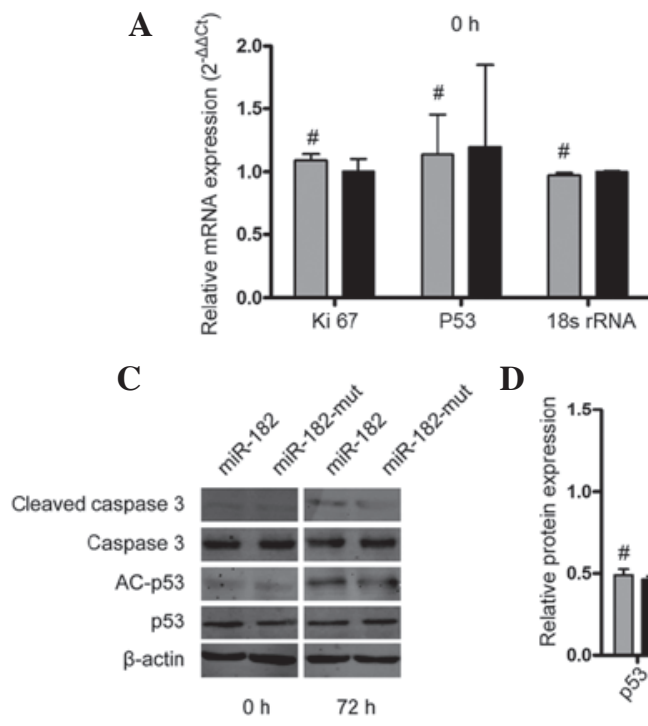

D

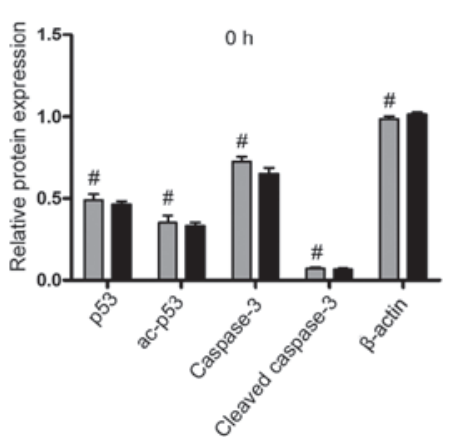

B

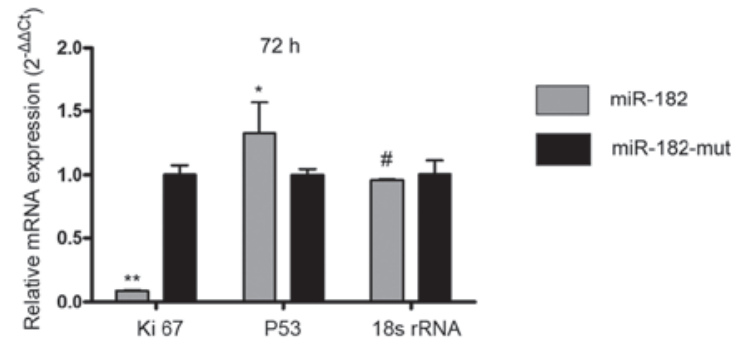

$\mathbf{E}$

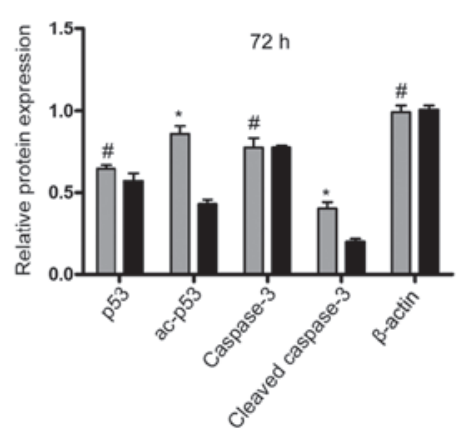

Figure 4. miR-182 expression promoted apoptosis in U251 cells via regulation of ki67 and p53. (A) and (B) Reverse transcription-quantitative polymerase chain reaction analysis, and (C), (D) and (E) western blot analysis. ${ }^{*} \mathrm{P}<0.05,{ }^{* *} \mathrm{P}<0.01$ and ${ }^{*} \mathrm{P}>0.05$ vs. mutant miR-182-transfected cells. $\mathrm{n}=3$. miR, microRNA; ac-p53, acetylated-p53.

expression of neuritin in the U251 cell line following miR-182 transfection influences cell cycle progression and reduces cell invasion and migration.
miR-182 inhibition of neuritin promotes apoptosis in the U251 cell line. In order to determine whether inhibition of neuritin by miR-182 influences cell proliferation and 
apoptosis in U251 cells, RT-qPCR and western blotting were performed. As shown in Fig. $4 \mathrm{~A}$ and $\mathrm{B}$, the expression of Ki-67 mRNA (23), a cell proliferation-related protein, was inhibited when the miR-182 expression plasmid was transfected into U251 cells after $72 \mathrm{~h}$, compared with that in the mutant miR-182-transfected cells. In addition, p53 mRNA levels were increased in miR-182-transfected cells, compared with levels in mutant miR-182-transfected cells. Western blotting demonstrated changes in the levels of apoptosis-related proteins. It was shown that the protein levels of cleaved caspase 3 and acetyled-p53 were increased significantly in miR-182-transfected cells, compared with levels in mutant miR-182-transfected cells at $72 \mathrm{~h}$ following transfection (Fig. 4C-E). These results indicated that exogenous miR-182 mediates inhibition of neuritin, suppresses cell proliferation and promotes apoptosis in astrocytoma cell lines.

\section{Discussion}

Astrocytomas are the most common type of glial tumors, and are associated with a poor prognosis due to infiltrative growth and a high migratory capacity $(24,25)$. The low median survival of patients with astrocytomas highlights the requirement for a comprehensive understanding of this disease.

Neuritin is a new member of the NF family, which has received little attention in terms of research into the development of astrocytoma from an epigenetic perspective. A previous study confirmed that neuritin is overexpressed in U251 cells and that its expression is positively correlated with tumor malignancy (16). The present study used a U251 cell line, transfected with an miR-182 vector, in order to detect whether the expression of neuritin is regulated by miR-182, and to examined its influence on cell proliferation and invasion in these cells.

To the best of our knowledge, the current results showed for the first time that miR-182 specifically targets neuritin. It was demonstrated that the mRNA and protein levels of neuritin were decreased in miR-182-transfected U251 cells compared with mutated miR-182-transfected cells. In addition, flow cytometry showed that the majority of the miR-182-transfected cells were arrested in the $\mathrm{S}$ phase of the cell cycle, suggesting that miR-182 overexpression influences cell cycle progression and tumor growth in vitro. Similarly, a transwell migration invasion assay demonstrated that the number of invading cells was significantly decreased in miR-182-transfected cells compared with mutant miR-182-transfected cells, suggesting that miR-182 suppresses cell invasion ability. Furthermore, RT-qPCR analysis showed that levels of Ki-67 and p53 mRNA, two important factors associated with cell proliferation and apoptosis, were decreased and increased, respectively, following transfection with miR-182. Western blotting also confirmed that the levels of two apoptosis-related proteins increased significantly when cells were transfected with miR-182, compared with transfection with mutant miR-182. These data demonstrate that miR-182 targets neuritin and regulates U251 cell proliferation and invasion. Inhibition or loss of miR-182 may lead to neuritin overexpression and thus promote tumorigenesis.
In conclusion, the present study showed that miR-182 specifically targets neuritin, and inhibits the proliferation and invasion of astrocytoma cells. Furthermore, miR-182-induced neuritin inhibition promoted apoptosis, which is a possible explanation for the observed suppression of cell proliferation and invasion. Further studies and experiments in vivo are required in order to fully explain the function of miR-182 and its potential targets in the development and progression of astrocytoma.

\section{Acknowledgements}

This study was supported by grants from the National Natural Science Foundation of China (grant nos. 81371410 and 81171205), the National Basic Research Program of China (973 Program; grant no. 2011CB707506), the National Natural Science Foundation of China (grant no. 81202811), Project funded by China Postdoctoral Science Foundation (grant no. 2014M550250) and Shanghai Municipal Health Bureau Fund (grant no. 20124320).

\section{References}

1. Wang W, Da R, Wang M, Wang T, Qi L, Jiang H, Chen W and $\mathrm{Li} \mathrm{Q}$ : Expression of brain-specific angiogenesis inhibitor 1 is inversely correlated with pathological grade, angiogenesis and peritumoral brain edema in human astrocytomas. Oncol Lett 5: 1513-1518, 2013.

2. Fuentes-Raspall R, Puig-Vives M, Guerra-Prio S, Perez-Bueno F and Marcos-Gragera R: Population-based survival analyses of central nervous system tumors from 1994 to 2008. An up-dated study in the temozolomide-era. Cancer Epidemiol 38: 244-247, 2014.

3. Ostrom QT, Bauchet L, Davis FG, Deltour I, Fisher JL, Langer CE, Pekmezci M, Schwartzbaum JA, Turner MC, Walsh KM, et al: The epidemiology of glioma in adults: A 'state of the science' review. Neuro Oncol 16: 2014.

4. Lee RC, Feinbaum RL and Ambros V: The C.elegans heterochronic gene lin-4 encodes small RNAs with antisense complementarity to lin-14. Cell 75: 843-854, 1993.

5. Lungu G, Stoica G and Ambrus A: MicroRNA profiling and the role of microRNA-132 in neurodegeneration using a rat model. Neurosci Lett 553: 153-158, 2013.

6. Cui J, Li D, Zhang W, Shen L and Xu X: Bioinformatics analyses combined microarray identify the deregulated microRNAs in oral cancer. Oncol Lett 8: 218-222, 2014.

7. Cheng W, Liu T, Wan X, Gao Y and Wang H: MicroRNA-199a targets CD44 to suppress the tumorigenicity and multidrug resistance of ovarian cancer-initiating cells. FEBS J 279: 2047-2059, 2012.

8. Kong W, Yang H, He L, Zhao JJ, Coppola D, Dalton WS and Cheng JQ: MicroRNA-155 is regulated by the transforming growth factor beta/Smad pathway and contributes to epithelial cell plasticity by targeting RhoA. Mol Cell Biol 28: 6773-6784, 2008.

9. Schetter AJ, Leung SY, Sohn JJv, Zanetti KA, Bowman ED, Yanaihara N, Yuen ST, Chan TL, Kwong DL, Au GK, et al: MicroRNA expression profiles associated with prognosis and therapeutic outcome in colon adenocarcinoma. Jama 299: 425-436, 2008.

10. Lanza G, Ferracin M, Gafà R, Veronese A, Spizzo R, Pichiorri F, Liu CG, Calin GA, Croce CM and Negrini M: mRNA/microRNA gene expression profile in microsatellite unstable colorectal cancer. Mol Cancer 6: 54, 2007.

11. Qin W, Ren Q, Liu T, Huang Y and Wang J: MicroRNA-155 is a novel suppressor of ovarian cancer-initiating cells that targets CLDN1. FEBS Lett 587: 1434-1439, 2013.

12. Huang S, He X, Ding J, Liang L, Zhao Y, Zhang Z, Yao X, Pan Z, Zhang P, Li J, et al: Upregulation of miR-23a approximately 27 a approximately 24 decreases transforming growth factor-beta-induced tumor-suppressive activities in human hepatocellular carcinoma cells. Int J Cancer 123: 972-978, 2008.

13. Zhou S and Zhou J: Neuritin, a neurotrophic factor in nervous system physiology. Curr Med Chem 21: 1212-1219, 2014. 
14. Raggo C, Ruhl R, McAllister S, Koon H, Dezube BJ, Früh K and Moses AV: Novel cellular genes essential for transformation of endothelial cells by Kaposi's sarcoma-associated herpesvirus. Cancer Res 65: 5084-5095, 2005.

15. Putz U, Harwell C and Nedivi E: Soluble CPG15 expressed during early development rescues cortical progenitors from apoptosis. Nat Neurosci 8: 322-331, 2005.

16. Zhang L, Zhao Y, Wang CG, Fei Z, Wang Y, Li L, Li L and Zhen HN: Neuritin expression and its relation with proliferation, apoptosis and angiogenesis in human astrocytoma. Med Oncol 28: 907-912, 2011.

17. Han D, Qin B, Liu G, Liu T, Ji G, Wu Y and Yu L: Characterization of neuritin as a novel angiogenic factor. Biochem Biophys Res Commun 415: 608-612, 2011.

18. Zito A, Cartelli D, Cappelletti G, Cariboni A, Andrews W, Parnavelas J, Poletti A and Galbiati M: Neuritin 1 promotes neuronal migration. Brain Struct Funct 219: 105-118, 2014.

19. Cheng W, Liu T, Jiang F, Liu C, Zhao X, Gao Y, Wang H and Liu Z: microRNA-155 regulates angiotensin II type 1 receptor expression in umbilical vein endothelial cells from severely pre-eclamptic pregnant women. Int J Mol Med 27: 393-399, 2011.
20. Zhang L, Liu T, Huang Y and Liu J: microRNA-182 inhibits the proliferation and invasion of human lung adenocarcinoma cells through its effect on human cortical actin-associated protein. Int J Mol Med 28: 381-388, 2011.

21. Liu T, Cheng W, Lai D, Huang Y and Guo L: Characterization of primary ovarian cancer cells in different culture systems. Oncol Rep 23: 1277-1284, 2010.

22. He M, Xu Z, Ding T, Kuang DM and Zheng L: MicroRNA-155 regulates inflammatory cytokine production in tumor-associated macrophages via targeting C/EBPbeta. Cell Mol Immunol 6: 343-352, 2009.

23. Jiang F, Liu T, He Y, Yan Q, Chen X, Wang H and Wan X: MiR-125b promotes proliferation and migration of type II endometrial carcinoma cells through targeting TP53INP1 tumor suppressor in vitro and in vivo. BMC Cancer 11: 425, 2011.

24. Liu Y, Tang K, Yan W, Wang Y, You G, Kang C, Jiang T and Zhang W: Identifying Ki-67 specific miRNA-mRNA interactions in malignant astrocytomas. Neurosci Lett 546: 36-41, 2013

25. Hamadi A, Giannone G, Takeda K and Rondé P: Glutamate involvement in calcium-dependent migration of astrocytoma cells. Cancer Cell Int 14: 42, 2014. 